\title{
Remote Desymmetrization at Near-Nanometer Group Separation Catalyzed by a Miniaturized Enzyme Mimic
}

\author{
Chad A. Lewis, ${ }^{\dagger}$ Anna Chiu, ${ }^{\ddagger}$ Michelle Kubryk, ${ }^{\ddagger}$ Jaume Balsells, ${ }^{\ddagger}$ David Pollard, ${ }^{\ddagger}$ Craig \\ K. Esser, ${ }^{\ddagger}$ Jerry Murry, ${ }^{\ddagger}$ Robert A. Reamer ${ }^{\ddagger}$ Karl B. Hansen ${ }^{\star \ddagger}$ and Scott J. Miller ${ }^{\star \dagger}$ \\ Department of Chemistry, Yale University, New Haven, Connecticut, 06511; Department of Chemistry, Boston College, \\ Chestnut Hill, Massachusetts, 02467-3860; Merck Research Laboratories, Rahway, New Jersey, 07065,
}

\section{Supplementary Materials}

General Procedures. Proton NMR spectra were recorded on either a Varian 400 or 300 $\mathrm{MHz}$ spectrometer. Proton chemical shifts are reported in $\mathrm{ppm}(\delta)$ relative to internal tetramethylsilane $(\delta 0.0)$. Data are reported as follows: chemical shift (multiplicity [singlet (s), doublet (d), triplet (t), quartet (q), and multiplet (m)], coupling constants $[\mathrm{Hz}]$, integration). Carbon NMR spectra were recorded on a Varian $400(100 \mathrm{MHz})$ spectrometer with complete proton decoupling. Carbon chemical shifts are reported in ppm $(\delta)$ relative to deuterated chloroform $\left(\delta\right.$ 77.16). NMR data were collected at $25^{\circ} \mathrm{C}$. Infrared spectra were obtained on a ThermoNicolet Avatar 210 spectometer. Analytical thin-layer chromatography (TLC) was performed using Silica Gel $60 \AA$ A F-254 precoated plates $(0.25 \mathrm{~mm}$ thickness). Visualization was accomplished by irradiation with a UV lamp and cerium ammonium molybdenate (CAM) solutions. Flash column chromatography was performed using Silica Gel $60 \AA$ (32-63 $\mu \mathrm{m})$. Optical rotations were recorded on a Rudolf Research Analytical Autopol IV Automatic polarimeter at the sodium D line (path length $50 \mathrm{~mm}$, corrected to $20.0^{\circ} \mathrm{C}$ ). High resolution mass spectra were obtained at the Mass Spectrometry Facility at the University of Illinois UrbanaChampaign. The method of ionization is given in parentheses.

Analytical and preparative reverse phase and normal phase HPLC were run on a Rainin SD-200 chromatograph equipped with a single wavelength UV detector $(214 \mathrm{~nm}$ or $254 \mathrm{~nm}$ ). Analytical normal phase HPLC was performed on a Hewlett-Packard 1100 Series chromatograph equipped with a diode array detector $(214 \mathrm{~nm}$ and $254 \mathrm{~nm})$. All reactions were carried out under an argon or nitrogen atmosphere employing oven or flame-dried glassware. Solvents were either used as received or purified according to standard procedures, and details are specified as necessary. Chloroform for catalytic runs was used as received, and typically contains $0.8 \% \mathrm{EtOH}$.

\section{Peptide Screens and Resulting Data}

The iterative procedure for screening the peptide-catalysts is shown below in SI-Figure 1. The most selective catalysts in each individual libraries are shown and the reaction conditions (absolute ee's are shown).

The amino acid appreviations beyond the most standard proteinogenic amino acids are as follows: Aib - $\alpha$-aminoisobutyric acid, Dbg - dibutyl glycine, Sp5 - 1aminocyclopentane-1-carboxylic acid, Sp6 - 1-aminocyclohexane-1-carboxylic acid, Pmh - $\pi$-methyl histidine, Tle - $\alpha$-tert-butyl glycine. 


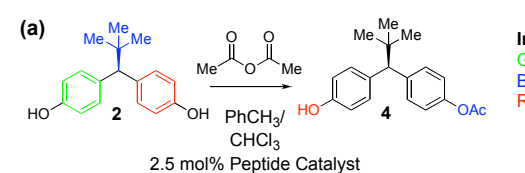

(c)

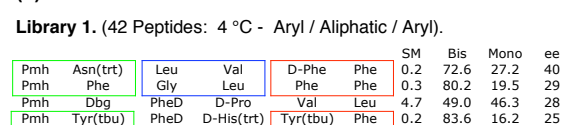

Library 2. (24 Peptides: $4^{\circ} \mathrm{C}$ - Optimization of Aliphatic Residues).

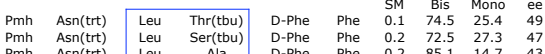

Library 3. (24 Peptides: $4{ }^{\circ} \mathrm{C}$ - Optimization of Aryl Residues).

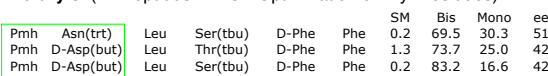

Library 4. (24 Peptides: $-20^{\circ} \mathrm{C}$ - Further Optimization of Residues)

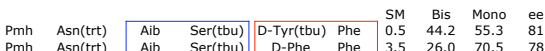

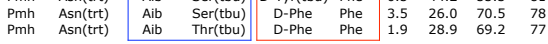

Library 5. (24 Peptides: $-20{ }^{\circ} \mathrm{C}$ - Further Optimization of Aryl Residues).

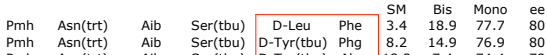

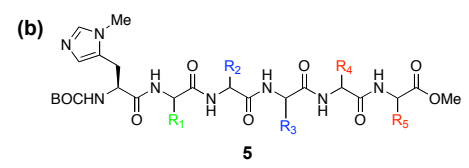

(d)

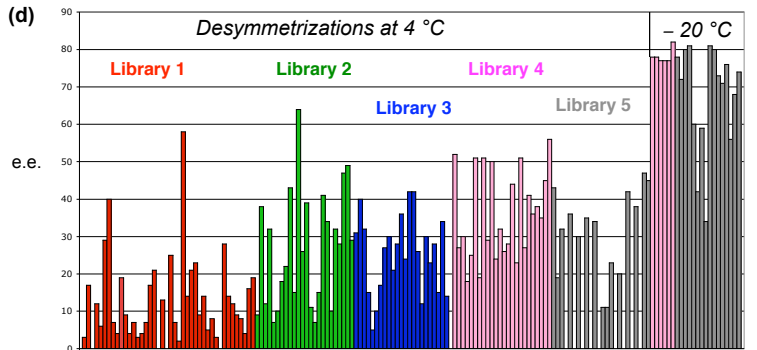

(e)

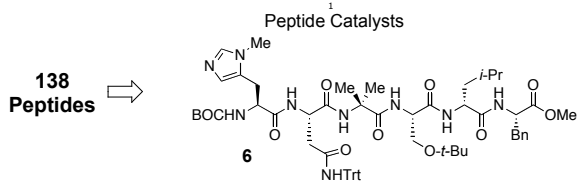

(f) Truncation study. (5 Peptides: - $20^{\circ} \mathrm{C}$ - Effect of Truncation).

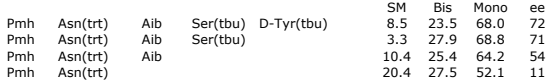

SI - Figure 1. (a) The reaction to be screened for the direct synthesis of monoacetate 4. (b) "Minimal" peptide-based catalyst library defined by structure 5. (c) Library-based catalyst optimization. Libraries were prepared that probed the aromatic-aliphatic-aromatic nature of the substrate according to the color coding in the Figure. Only representative library members are listed. (d) Histogram of complete set of peptide catalysts that were screened. (e) Structure of catalyst 6 that emerged from the fifth generation library. (f) Sample of truncated peptides that were screened to probe catalyst SAR.

\section{Peptide Synthesis and Screens}

Peptides were synthesized on solid support using commercially available Wang polystyrene resin preloaded with FMOC protected amino acid. Couplings were performed using 5 equiv amino acid derivative, 5 equiv HBTU, and 10 equiv Hünig's base in DMF, for $3 \mathrm{~h}$. Deprotections were performed using 20\% piperidine in DMF for 20 min (to minimize diketopiperazine formation; dipeptides were deprotected using $50 \%$ piperidine in DMF for $5 \mathrm{~min}$ ). Peptides were cleaved from solid support using a mixture of MeOH:DMF: $\mathrm{NEt}_{3}$ (9:1:1) for $5 \mathrm{~d}$. The peptides were characterized by electrospray mass spectrometry and used in parallel reaction screens without further purification. Peptide sequences, which proved selective for the desymmeterizations, were purified by reverse phase HPLC techniques. Preparative HPLC was performed using a reverse phase RP-18 X Terra (Waters) column, eluting with $60-70 \%$ methanol in water with a gradient over 25 minutes, at a flow rate of $4 \mathrm{~mL} / \mathrm{min}$.

Peptide 14.

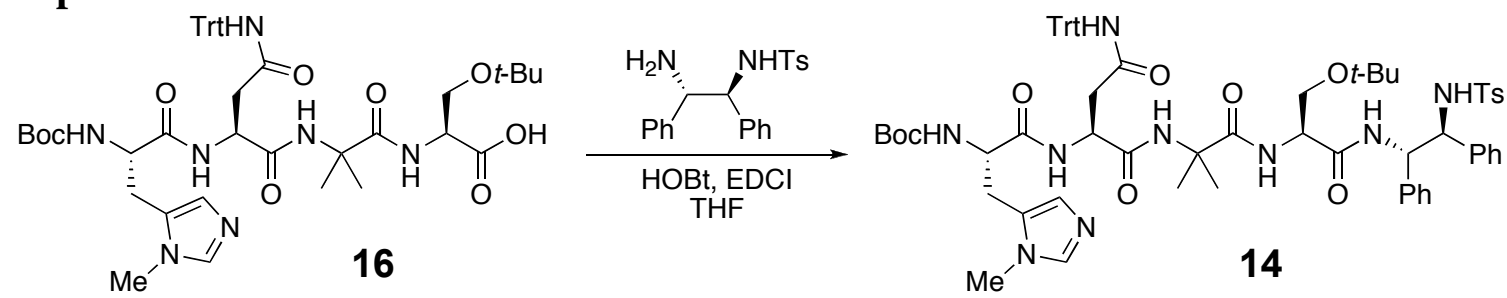

Tetrapeptide-acid 16 was synthesized using standard solution phase peptide synthesis techniques. ${ }^{1}$ To a solution of tetrapeptide acid $\mathbf{1 6}(2.60 \mathrm{~g}, 3.00 \mathrm{mmol})$ in $86.0 \mathrm{~mL}$ of THF

${ }^{1}$ Bodanszky, M.; Bodanszky, A. The Practice of Peptide Synthesis; Springer-Verlag: Berlin, 1993. 
at $0{ }^{\circ} \mathrm{C}$ was added (1S, 2S)-(+)- $N$-p-Tosyl-1,2-diphenylethylenediamine $(1.10 \mathrm{~g}, 3.00$ $\mathrm{mmol})$, HOBt $(580 \mathrm{mg}, 4.30 \mathrm{mmol})$, and EDCI $(700 \mathrm{mg}, 3.60 \mathrm{mmol})$. The solution was stirred for 3 hours and diluted with water. The biphasic solution was separated and the aqueous solution was extracted with EtOAc $(3 \times 50 \mathrm{~mL})$. The combined organic extracts were dried over $\mathrm{Na}_{2} \mathrm{SO}_{4}$, filtered, and concentrated in vacuo. The residue was purified by flash chromatography $\left(3 \% \mathrm{MeOH} / \mathrm{CH}_{2} \mathrm{Cl}_{2}\right)$ to afford $3.50 \mathrm{~g}(2.90 \mathrm{mmol}, 97 \%)$ of tetrapeptide amide $\mathbf{1 4}$ as a white solid. $[\alpha]_{\mathrm{D}}^{20.0}-37.3\left(c 0.33, \mathrm{CHCl}_{3}\right) ;{ }^{1} \mathbf{H} \mathbf{~ N M R}(600.133$ $\left.\mathrm{MHz}, \mathrm{CDCl}_{3}\right) \delta 8.20(\mathrm{~d}, J=6.2 \mathrm{~Hz}, 1 \mathrm{H}), 8.09(\mathrm{~d}, J=10.1 \mathrm{~Hz}, 1 \mathrm{H}), 8.05(\mathrm{~s}, 1 \mathrm{H}), 7.69$ (s, $1 \mathrm{H}), 7.61(\mathrm{~s}, 1 \mathrm{H}), 7.49(\mathrm{~m}, 3 \mathrm{H}), 7.27(\mathrm{~m}, 2 \mathrm{H}), 7.23-7.17(\mathrm{~m}, 9 \mathrm{H}), 7.13-7.07(\mathrm{~m}, 15 \mathrm{H})$, $6.92(\mathrm{~s}, 1 \mathrm{H}), 6.68(\mathrm{~d}, J=8.0 \mathrm{~Hz}, 2 \mathrm{H}), 5.42\left(\mathrm{dd}, J_{l}=10.2, J_{2}=3.6 \mathrm{~Hz}, 1 \mathrm{H}\right), 5.12-5.11(\mathrm{~m}$, $2 \mathrm{H}), 4.47(\mathrm{~m}, 1 \mathrm{H}), 4.37\left(\mathrm{dd}, J_{1}=6.0, J_{2}=3.0 \mathrm{~Hz}, 1 \mathrm{H}\right), 3.87\left(\mathrm{dd}, J_{1}=7.6, J_{2}=1.0 \mathrm{~Hz}\right.$, $1 \mathrm{H}), 3.61(\mathrm{~s}, 3 \mathrm{H}), 3.36\left(\mathrm{dd}, J_{1}=9.0, J_{2}=3.6 \mathrm{~Hz}, 1 \mathrm{H}\right), 3.17\left(\mathrm{dd}, J_{1}=15.6, J_{2}=4.2 \mathrm{~Hz}\right.$, $1 \mathrm{H}), 3.13\left(\mathrm{dd}, J_{1}=15.6, J_{2}=6.6 \mathrm{~Hz}, 1 \mathrm{H}\right), 2.86\left(\mathrm{dd}, J_{1}=15.6, J_{2}=10.2 \mathrm{~Hz}, 1 \mathrm{H}\right), 2.65(\mathrm{dd}$, $\left.J_{1}=15.6, J_{2}=3.6 \mathrm{~Hz}, 1 \mathrm{H}\right), 2.18(\mathrm{~s}, 3 \mathrm{H}), 1.66(\mathrm{~s}, 3 \mathrm{H}), 1.54(\mathrm{~s}, 3 \mathrm{H}), 1.32(\mathrm{~s}, 9 \mathrm{H}), 1.13(\mathrm{~s}$, 9H); ${ }^{13} \mathbf{C}$ NMR $\left(125.77 \mathrm{MHz}, \mathrm{CDCl}_{3}\right) \delta 173.2,171.1,170.2,170.0,156.5,144.4,142.1$, 139.2 , 139.0, 137.9, 137.1, 128.8, 128.7, 128.4, 128.3, 127.9, 127.7, 127.3, 127.0, 126.9, $126.8,126.3,126.1,81.8,75.5,70.8,61.7,61.1,57.9,57.1,54.8,53.8,51.4,36.1,31.4$, 28.1, 27.2, 26.9, 26.1, 26.0, 23.3, 21.3. HRMS (ESI) calcd. for $\mathrm{C}_{67} \mathrm{H}_{79} \mathrm{~N}_{9} \mathrm{O}_{10} \mathrm{~S}(\mathrm{M}+\mathrm{H})$ 1202.5748, found 1202.5741 .

\section{Optimized Desymmetrization Procedure}

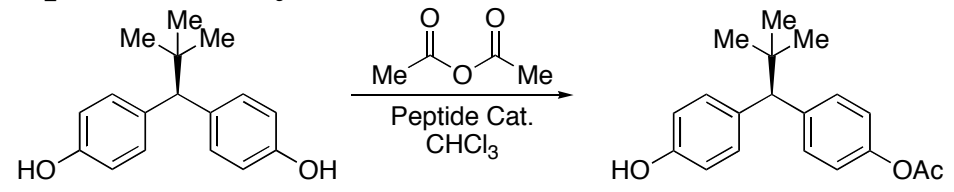

To a solution of bisphenol $2(90.0 \mathrm{mg}, 351.6 \mu \mathrm{mol})$ in $15.0 \mathrm{~mL}$ of $\mathrm{CHCl}_{3}$ at $-30{ }^{\circ} \mathrm{C}$ was added catalyst $14(21.0 \mathrm{mg}, 17.46 \mu \mathrm{mol})$ followed by acetic anhydride $(70.0 \mu \mathrm{L}, 740.5$ $\mu \mathrm{mol})$. The solution was stirred for 20 hours and quenched by the addition of $2 \mathrm{~mL}$ of $\mathrm{MeOH}$. Upon warming to room temperature, EtOAc and water was added. The biphasic solution was separated and the aqueous solution was extracted with EtOAc $(2 \times 10 \mathrm{~mL})$. The combined organic extracts were dried over $\mathrm{Na}_{2} \mathrm{SO}_{4}$, filtered, and concentrated in vacuo. The residue was purified by flash chromatography $(25 \% \mathrm{EtOAc/hexanes)}$ to afford $84.0 \mathrm{mg}(281.5 \mu \mathrm{mol}, 80 \%)$ of monoacetate 4 and $25.0 \mathrm{mg}(73.44 \mu \mathrm{mol}, 20 \%)$ of bisacetate phenol 3 .

\section{(R)-4-(1-(4-Hydroxyphenyl)-2,2-tert-butyl)phenyl acetate 4.

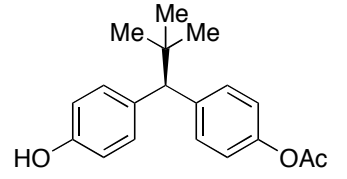

$[\alpha]_{\mathrm{D}}^{20.0}+12.7\left(c\right.$ 0.22, $\left.\mathrm{CHCl}_{3}\right)$; M.p.: $129{ }^{\circ} \mathrm{C} ;{ }^{1} \mathbf{H}$ NMR $\left(\mathrm{CDCl}_{3}, 400 \mathrm{MHz}\right) \delta 7.37(\mathrm{~d}, J=$ $8.4 \mathrm{~Hz}, 2 \mathrm{H}), 7.20(\mathrm{~d}, J=8.4 \mathrm{~Hz}, 2 \mathrm{H}), 6.97(\mathrm{~d}, J=8.4 \mathrm{~Hz}, 2 \mathrm{H}), 6.66(\mathrm{~d}, J=8.4 \mathrm{~Hz}, 2 \mathrm{H})$, 5.83 (br s, 1H), 3.62 (s, 1H), 2.23 (s, 3H), 0.96 (s, 9H); ${ }^{13} \mathbf{C ~ N M R ~}\left(\mathrm{CDCl}_{3}, 100 \mathrm{MHz}\right) \delta$ $170.1,153.9,148.6,141.1,134.8,130.7,130.5,120.7,114.8,62.9,35.2,29.4,21.4$; IR (film, $\mathrm{cm}^{-1}$ ) 3402, 2967, 2911, 2873, 1740, 1614, 1520, 1369, 1206, 1111, 1016, 908, 853, 733, 563; TLC R f $_{\mathrm{f}} 0.67$ (1/1 Hexanes/EtOAc v/v); HPLC 95\% ee, Chiral HPLC 
utilized a Chiralpak AD (Daicel, $0.46 \mathrm{~cm} \times 15 \mathrm{~cm}, 20{ }^{\circ} \mathrm{C}$ ) eluting at $0.75 \mathrm{~mL} / \mathrm{min}$ with $75 \%$ hexane/isopropanol. Retention times: $\mathrm{R}_{T(\mathrm{~S})}=5.5 \mathrm{~min} ; \mathrm{R}_{T(\mathrm{R})}=6.2 \mathrm{~min} ; \mathbf{H R M S}\left(\mathrm{EI}^{+}\right)$ $m / z$ Calc'd for $\mathrm{C}_{19} \mathrm{H}_{22} \mathrm{O}_{3} 298.1569$, found 298.1574.

\section{4,4'-(2,2-dimethylpropane-1,1-diyl)bis(4,1-phenylene) diacetate 3.}

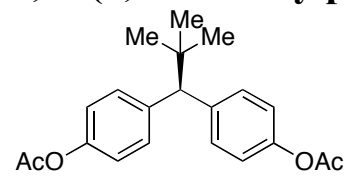

M.p.: $131-132{ }^{\circ} \mathrm{C} ;{ }^{1} \mathbf{H}$ NMR $\left(\mathrm{CDCl}_{3}, 400 \mathrm{MHz}\right) \delta 7.39\left(\mathrm{dd}, J_{1}=6.8 \mathrm{~Hz}, J_{2}=1.6 \mathrm{~Hz}\right.$, $4 \mathrm{H}), 6.99\left(\mathrm{dd}, J_{1}=6.8 \mathrm{~Hz}, J_{2}=1.6 \mathrm{~Hz}, 4 \mathrm{H}\right), 3.72(\mathrm{~s}, 1 \mathrm{H}), 2.22(\mathrm{~s}, 6 \mathrm{H}), 1.00(\mathrm{~s}, 9 \mathrm{H}) ;{ }^{13} \mathrm{C}$ NMR $\left(\mathrm{CDCl}_{3}, 100 \mathrm{MHz}\right) \delta 169.1,148.8,140.2,130.5,120.8,63.1,35.2,29.3,21.3$; IR (film, $\mathrm{cm}^{-1}$ ) 2967, 2911, 2873, 1759, 1520, 1381, 1211, 1016, 916, 859, 739, 570; TLC $\mathrm{R}_{\mathrm{f}}=0.74(1 / 1$ Hexanes/EtOAc v/v) HPLC Chiral HPLC utilized a Chiralpak AD (Daicel, $0.46 \mathrm{~cm} \times 15 \mathrm{~cm}, 20{ }^{\circ} \mathrm{C}$ ) eluting at $0.75 \mathrm{~mL} / \mathrm{min}$ with $75 \%$ hexane/isopropanol. Retention time: $4.0 \mathrm{~min}$; HRMS $\left(\mathrm{EI}^{+}\right) \mathrm{m} / \mathrm{z}$ Calc'd for $\mathrm{C}_{21} \mathrm{H}_{24} \mathrm{O}_{4} 340.1675$, found 340.1681 .

Typical Chiral HPLC trace for a.) racemic monoacetate 4 and bisacetate 3. b.) purified sample of $\mathbf{3}$ from peptide-catalyzed acylation.

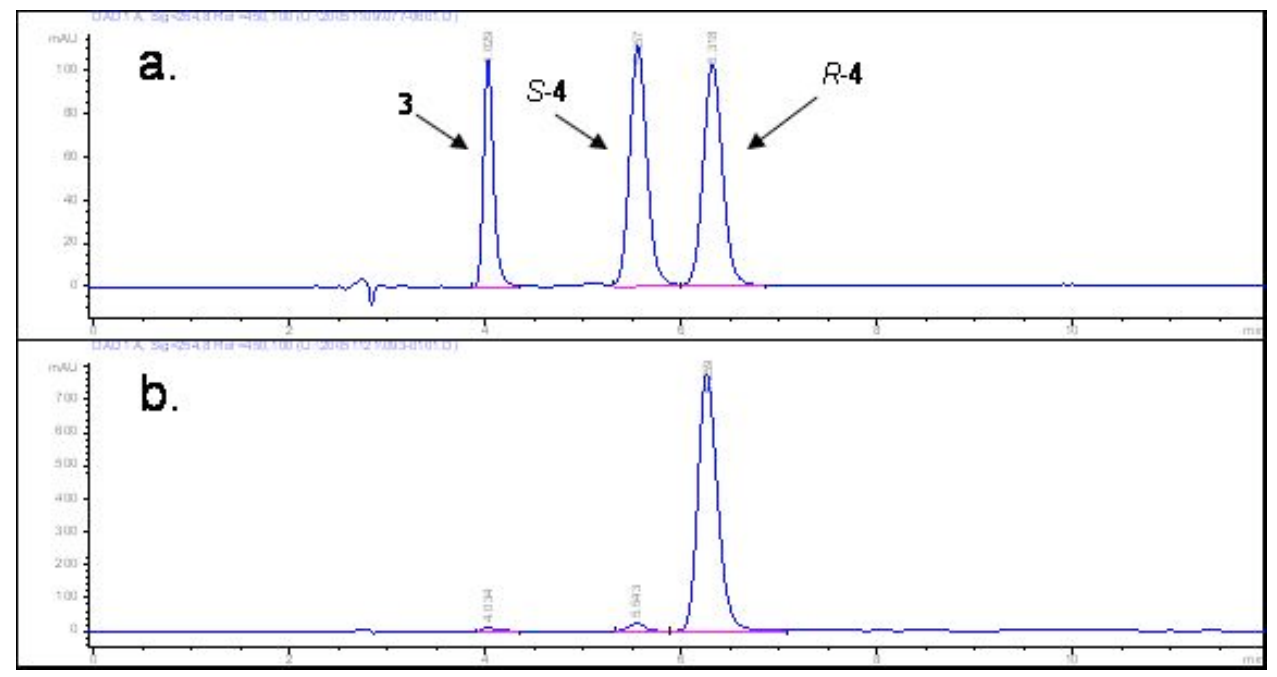




\section{Additional Peptide Catalyst Development Studies - Catalyst Loading and Concentration.}

(MK-0744 is the same as catalyst 14 in the text.)

The effect of catalyst loading $(0.25 \mathrm{~mol} \%, 2 \mathrm{~mol} \%$, and $5 \mathrm{~mol} \%)$ and concentration $(0.025 \mathrm{M}, 0.1 \mathrm{M}, 0.2 \mathrm{M})$ for the desymmetrization of bisphenol 2 was examined. At very low catalyst loading, poor enantioselectivity and reaction conversion was observed and the effect of added THF was minimal (entry 1-3). At $2-2.5 \mathrm{~mol} \%$ catalyst loading (standard conditions used previously for the screening of other catalyst analogs), the combination of increased concentration and $\mathrm{THF} / \mathrm{CHCl}_{3}$ ratio lowered the ee\% (entry 6). At $5 \mathrm{~mol} \%$ catalyst loading, good enantioselectivity (91\% ee) was observed at high dilution and low concentration of THF (entry 7). Upon re-screening with recrystallized starting material, the enantioselectivity under these conditions was $95 \%$ ee, and this result was found to be highly reproducible.

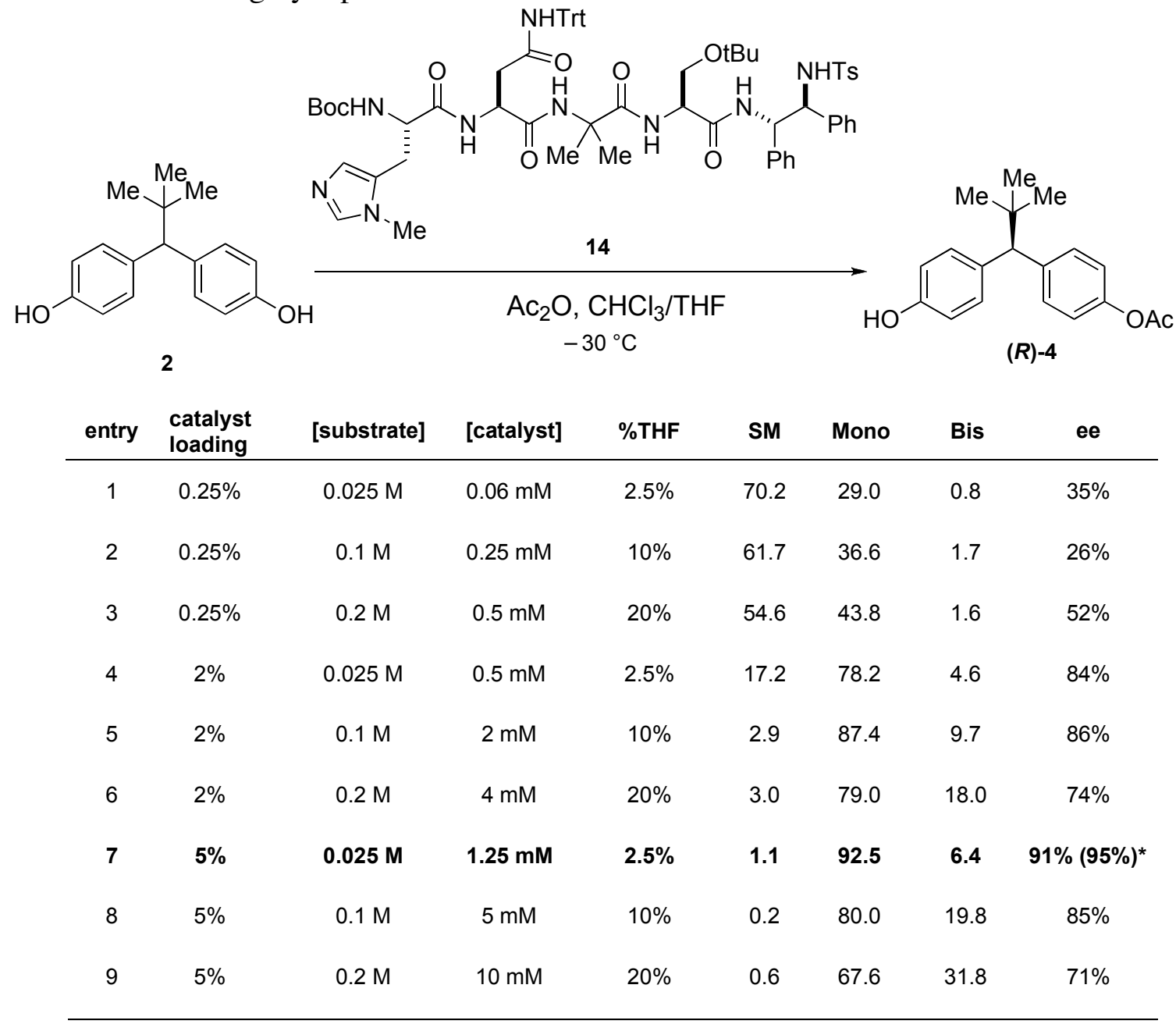

* The results were optimized with freshly recrystallized starting material (2). 
From the results above, the best enantioselection was obtained with a catalyst concentration of $1.25 \mathrm{mM}$ with minimal THF in the reaction. Several more conditions were screened, which are summarized below. In the absence of THF, reaction under the same conditions as entry 10 gave a slight increase in ee\% (96\% ee). Entry 12, which uses $2 \mathrm{~mol} \%$ of the peptide catalyst and $0.06 \mathrm{M}$ concentration in substrate, provided the monoacetate in $94 \%$ ee. Unfortunately, peptide loading of $1 \mathrm{~mol} \%$ resulted in a significant decrease in ee\% (entry 13).

\begin{tabular}{ccccccccc} 
entry & $\begin{array}{c}\text { catalyst } \\
\text { loading }\end{array}$ & [substrate] & [catalyst] & \%THF & SM & Mono & Bis & ee \\
\hline 10 & $5 \%$ & $0.025 \mathrm{M}$ & $1.25 \mathrm{mM}$ & $2.5 \%$ & 0 & 92.2 & 7.8 & 94.5 \\
11 & $5 \%$ & $0.025 \mathrm{M}$ & $1.25 \mathrm{mM}$ & $0 \%$ & 0 & 90.8 & 9.2 & 95.9 \\
12 & $2 \%$ & $0.06 \mathrm{M}$ & $1.25 \mathrm{mM}$ & $2.5 \%$ & 3.1 & 91.2 & 5.7 & 93.9 \\
13 & $1 \%$ & $0.12 \mathrm{M}$ & $1.25 \mathrm{mM}$ & $2.5 \%$ & 17.2 & 77.8 & 5.1 & 87.9
\end{tabular}

\section{NMR Studies of Peptide-Substrate Complexation.}

The peptide catalyst 14 was dissolved in $\mathrm{CDCl}_{3}$ at the concentration of the reaction conditions. EtOH was introduced to an approximate concentration 0.8\%. Acetic acid was then introduced to mimic the reaction medium after $\sim 4-5$ turnovers of the catalytic cycle. The ${ }^{1} \mathrm{H}$ NMR spectrum was then recorded. Subsequently, substrate 2 was introduced $\left(20 \mathrm{~mol} \%\right.$ ), and the ${ }^{1} \mathrm{H}$ NMR spectrum was recorded again after several minutes of medium equilibration. The stacked plots are shown in the manuscript text. 

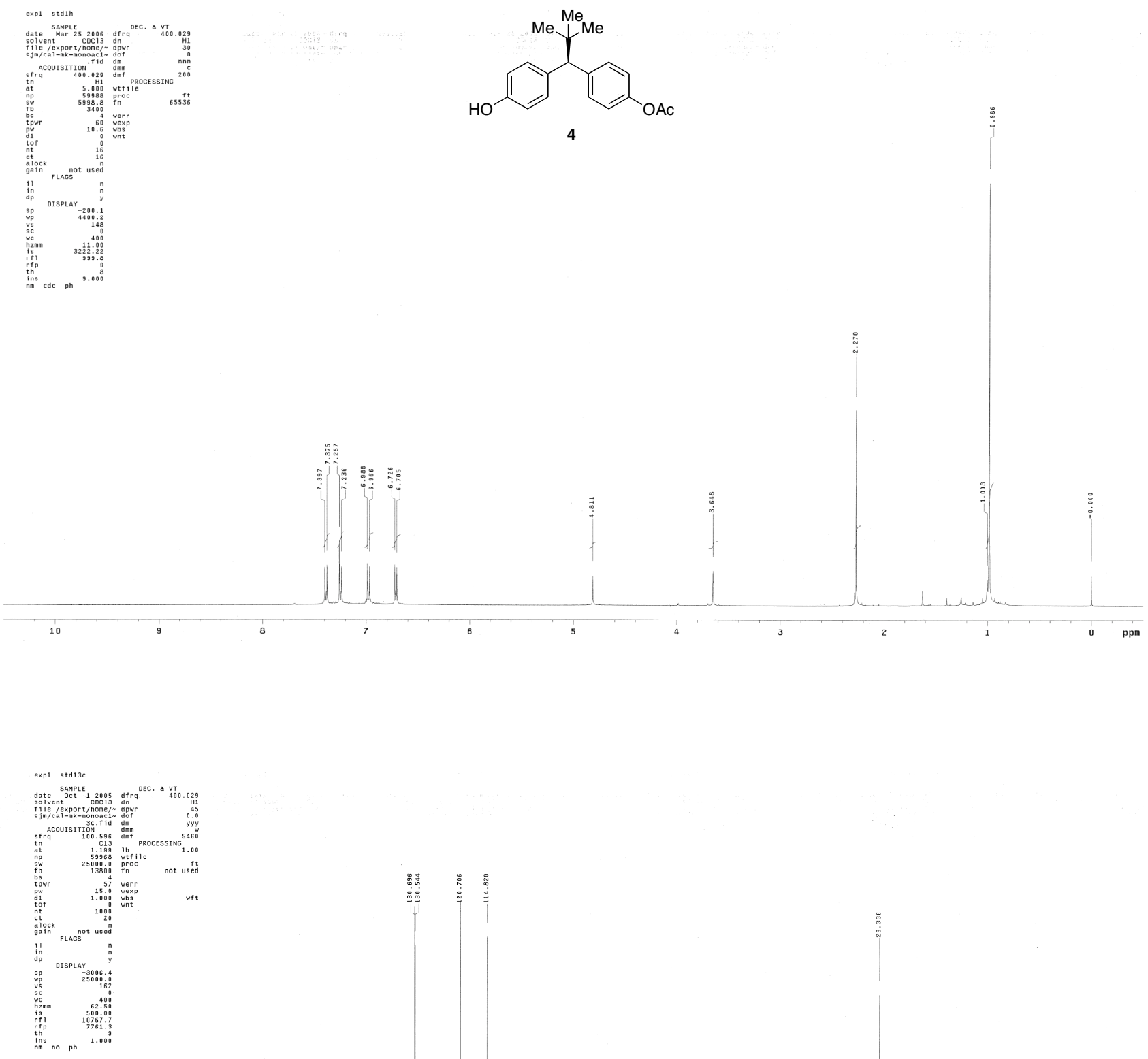

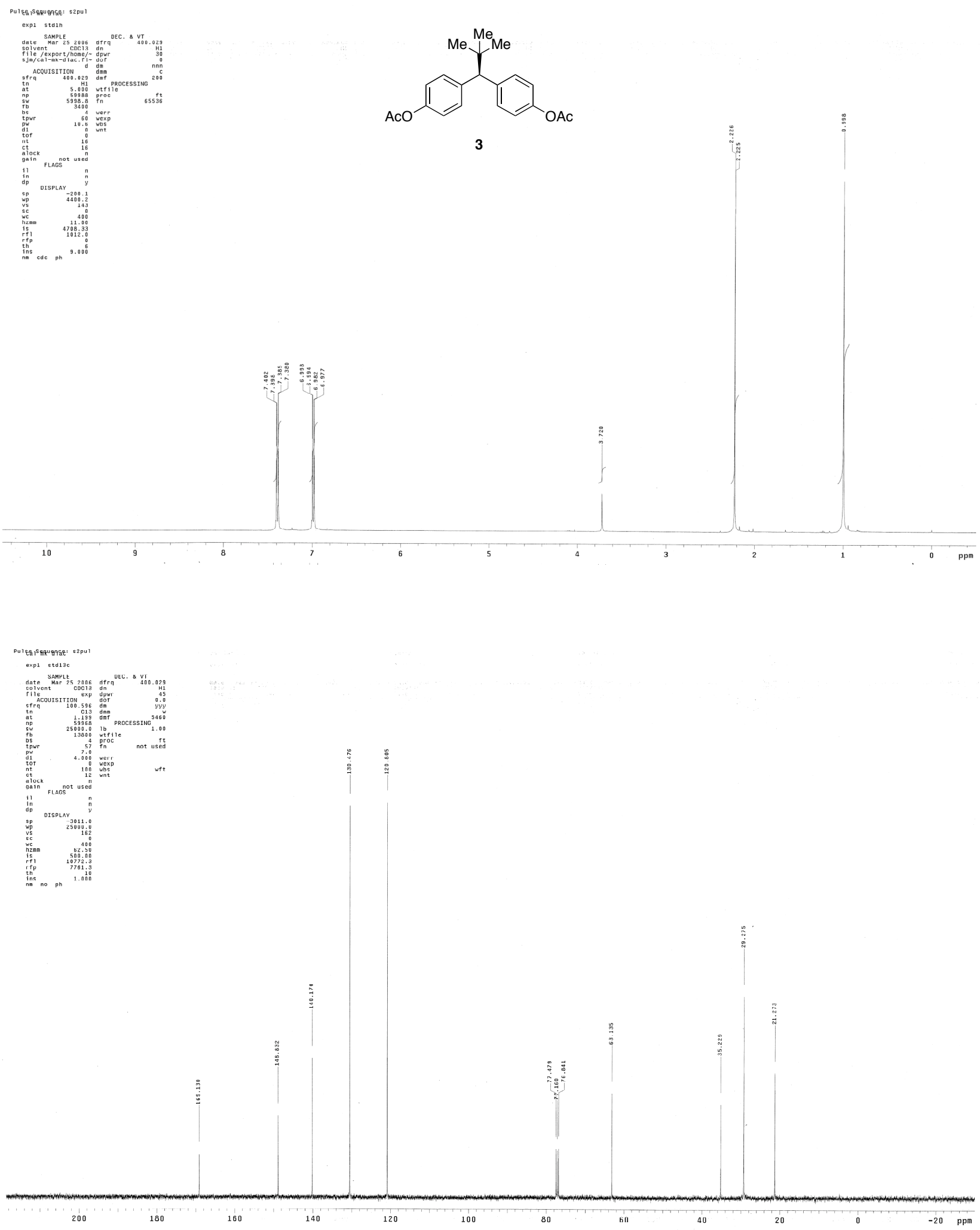
Table 1.

\begin{tabular}{|c|c|c|c|c|}
\hline Peptide & Sequence & Library & $\begin{array}{c}\text { Temp } \\
\left({ }^{\circ} \mathrm{C}\right)\end{array}$ & $\%$ ee \\
\hline $1 \mathrm{~A}$ & BOC-Pmh-Asp(OBut)-Trp-Aib-Tyr(Bn)-Phe-OMe & 1 & 4 & 3 \\
\hline 1B & BOC-Pmh-Asp(OBut)-Asn-Aib-Phe-Phe-OMe & 1 & 4 & -17 \\
\hline $1 \mathrm{C}$ & BOC-Pmh-Pro-Trp-Arg(BOC)2-Tyr(OH)-Phe-OMe & 1 & 4 & 0 \\
\hline 1D & BOC-Pmh-Tyr-Phe-His(Trt)-Tyr(tbu)-Phe-OMe & 1 & 4 & -12 \\
\hline $1 \mathrm{E}$ & BOC-Pmh-Pro-Asn-His-Tyr(OH)-Phe-OMe & 1 & 4 & -6 \\
\hline $1 \mathrm{~F}$ & BOC-Pmh-Phe-Gly-Leu-Phe-Phe-OMe & 1 & 4 & -29 \\
\hline $1 \mathrm{G}$ & BOC-Pmh-Asn(trt)-Leu-Val-PheD-Phe-OMe & 1 & 4 & -40 \\
\hline $1 \mathrm{H}$ & BOC-Pmh-Asp(OBut)-Tyr(Bn)-Aib-Met-Phe-OMe & 1 & 4 & 7 \\
\hline 1I & $\begin{array}{c}\text { BOC-Pmh-Leu-Tyr(tbu)-Arg(BOC)2-Lys(BOC)-Phe- } \\
\text { OMe }\end{array}$ & 1 & 4 & 4 \\
\hline $1 \mathrm{~J}$ & BOC-Pmh-Ile-Val-Asn-Thr(tbu)-Phe-OMe & 1 & 4 & -19 \\
\hline $1 \mathrm{~K}$ & BOC-Pmh-Glu-Val-Phe-Ala-Phe-OMe & 1 & 4 & -9 \\
\hline $1 \mathrm{~L}$ & BOC-Pmh-Trp-His-Gly-Phe-Phe-OMe & 1 & 4 & -4 \\
\hline $1 \mathrm{M}$ & BOC-Pmh-Phe-Gly-VaID-AlaD-Phe-OMe & 1 & 4 & -7 \\
\hline $1 \mathrm{~N}$ & BOC-Pmh-Aib-Pro-Phe-ValD-Phe-OMe & 1 & 4 & 3 \\
\hline 10 & BOC-Pmh-Pro-Aib-Pro-Tyr(Bn)-Phe-OMe & 1 & 4 & -4 \\
\hline $1 \mathrm{P}$ & BOC-Pmh-PheD-Pro-Asn(trt)-Val-Phe-OMe & 1 & 4 & -7 \\
\hline 1Q & BOC-Pmh-Phe-PheD-Pro-Phe-Phe-OMe & 1 & 4 & -17 \\
\hline $1 \mathrm{R}$ & BOC-Pmh-Phe-Gly-Pro-Phe-Phe-OMe & 1 & 4 & -21 \\
\hline $1 \mathrm{~S}$ & BOC-Pmh-Thr(tbu)-ValD-His(trt)-PheD-Phe-OMe & 1 & 4 & 0 \\
\hline $1 \mathrm{~T}$ & BOC-Pmh-Ser(tbu)-AlaD-PheD-His(trt)-Phe-OMe & 1 & 4 & -13 \\
\hline $1 \mathrm{U}$ & $\begin{array}{l}\text { BOC-Pmh-Tyr(tbu)-PheD-HisD(trt)-Tyr(tbu)-Phe- } \\
\text { OMe }\end{array}$ & 1 & 4 & -25 \\
\hline $1 \mathrm{~V}$ & BOC-Pmh-Val-Glu(tbu)-Asp(OBut)-PheD-Phe-OMe & 1 & 4 & -7 \\
\hline $1 \mathrm{~W}$ & $\begin{array}{c}\text { BOC-Pmh-LeuD-Tyr(tbu)-Arg(BOC)2-Lys(BOC)-Phe- } \\
\text { OMe }\end{array}$ & 1 & 4 & 2 \\
\hline $1 \mathrm{X}$ & BOC-Pmh-IleD-ValD-Asn-Thr(tbu)-Phe-OMe & 1 & 4 & 58 \\
\hline $1 \mathrm{Y}$ & BOC-Pmh-Pro-His(trt)-Phe-ValD-Phe-OMe & 1 & 4 & 14 \\
\hline $1 \mathrm{Z}$ & BOC-Pmh-Thr(tbu)-ProD-Ser(tbu)-Phe-Phe-OMe & 1 & 4 & -21 \\
\hline $2 \mathrm{~A}$ & BOC-Pmh-Leu-Aib-Phe-ValD-Phe-OMe & 1 & 4 & -23 \\
\hline 2B & $\begin{array}{l}\text { BOC-Pmh-Asp(OBut)-Asn(trt)-Arg(BOC)2-Tyr(OH)- } \\
\text { Phe-OMe }\end{array}$ & 1 & 4 & -9 \\
\hline $2 \mathrm{C}$ & $\begin{array}{l}\text { BOC-Pmh-Asp(OBut)-Asn(trt)-Asn(trt)-Tyr(tbu)-Phe- } \\
\text { OMe }\end{array}$ & 1 & 4 & -14 \\
\hline $2 \mathrm{D}$ & $\begin{array}{l}\text { BOC-Pmh-Asp(OBut)-Asn(trt)-His(trt)-Tyr(Bn)-Phe- } \\
\text { OMe }\end{array}$ & 1 & 4 & -5 \\
\hline $2 \mathrm{E}$ & BOC-Pmh-Asp(OBut)-Asn-Met-Tyr(OH)-Phe-OMe & 1 & 4 & -8 \\
\hline $2 \mathrm{~F}$ & BOC-Pmh-Thr(tbu)-Asn(trt)-Arg-Tyr(Bn)-Phe-OMe & 1 & 4 & -3 \\
\hline $2 \mathrm{G}$ & BOC-Pmh-Ser(tbu)-Asn(trt)-Arg-Tyr(Bn)-Phe-OMe & 1 & 4 & 0 \\
\hline $2 \mathrm{H}$ & BOC-Pmh-Dbg-PheD-ProD-Val-Leu-OMe & 1 & 4 & -28 \\
\hline $2 \mathrm{I}$ & BOC-Pmh-Dbg-Phe-Pro-ValD-Leu-OMe & 1 & 4 & -14 \\
\hline $2 \mathrm{~J}$ & BOC-Pmh-Ala-His(trt)-Tyr(Bn)-Leu-Ile-OMe & 1 & 4 & -12 \\
\hline
\end{tabular}




\begin{tabular}{|c|c|c|c|c|}
\hline $2 \mathrm{~K}$ & BOC-Pmh-Ile-Phe-Leu-Phe-Ile-OMe & 1 & 4 & -9 \\
\hline $2 \mathrm{~L}$ & BOC-Pmh-Hyp(OBut)-Thr(tbu)-AlaD-Val-Ile-OMe & 1 & 4 & -8 \\
\hline $2 \mathrm{M}$ & BOC-Pmh-Phe-Gly-Tyr(tbu)-Phe-Ile-OMe & 1 & 4 & -4 \\
\hline $2 \mathrm{~N}$ & BOC-Pmh-Tyr(tbu)-Val-Cha-PheD-Ile-OMe & 1 & 4 & -16 \\
\hline $2 \mathrm{O}$ & $\begin{array}{c}\text { BOC-Pmh-Asp(OBut)-Asn(trt)-Lys(BOC)-Tyr(OH)-Ile- } \\
\text { OMe }\end{array}$ & 1 & 4 & -19 \\
\hline $2 \mathrm{P}$ & BOC-Pmh-Asn(trt)-Leu-Val-Phe-PheD-OMe & 2 & 4 & -9 \\
\hline $2 \mathrm{Q}$ & BOC-Pmh-Asn(trt)-Leu-Val-PheD-PheD-OMe & 2 & 4 & -38 \\
\hline $2 \mathrm{R}$ & BOC-Pmh-Asn(trt)-Ile-Val-PheD-Phe-OMe & 2 & 4 & -12 \\
\hline $2 \mathrm{~S}$ & BOC-Pmh-Asn(trt)-Ala-Val-PheD-Phe-OMe & 2 & 4 & -32 \\
\hline $2 \mathrm{~T}$ & BOC-Pmh-Asn(trt)-AlaD-Val-PheD-Phe-OMe & 2 & 4 & -7 \\
\hline $2 \mathrm{U}$ & BOC-Pmh-Asn(trt)-Val-Val-PheD-Phe-OMe & 2 & 4 & -10 \\
\hline $2 \mathrm{~V}$ & BOC-Pmh-Asn(trt)-ValD-Val-PheD-Phe-OMe & 2 & 4 & -18 \\
\hline $2 \mathrm{~W}$ & BOC-Pmh-Asn(trt)-Leu-Ile-PheD-Phe-OMe & 2 & 4 & -22 \\
\hline $2 \mathrm{X}$ & BOC-Pmh-Asn(trt)-Leu-Ala-PheD-Phe-OMe & 2 & 4 & -43 \\
\hline $2 \mathrm{X}$ & BOC-Pmh-Asn(trt)-Leu-Ala-PheD-Phe-OMe & 2 & -20 & -59 \\
\hline $2 \mathrm{Y}$ & BOC-Pmh-Asn(trt)-Leu-AlaD-PheD-Phe-OMe & 2 & 4 & 15 \\
\hline $2 Z$ & BOC-Pmh-Asn(trt)-Leu-ValD-PheD-Phe-OMe & 2 & 4 & 64 \\
\hline $2 Z$ & BOC-Pmh-Asn(trt)-Leu-ValD-PheD-Phe-OMe & 2 & -20 & -10 \\
\hline $3 \mathrm{~A}$ & BOC-Pmh-Asn(trt)-Tle-Val-PheD-Phe-OMe & 2 & 4 & -26 \\
\hline 3B & BOC-Pmh-Asn(trt)-Dbg-Val-PheD-Phe-OMe & 2 & 4 & -39 \\
\hline $3 \mathrm{C}$ & BOC-Pmh-Asn(trt)-Gly-Val-PheD-Phe-OMe & 2 & 4 & -11 \\
\hline $3 \mathrm{D}$ & BOC-Pmh-Asn(trt)-Leu-Dbg-PheD-Phe-OMe & 2 & 4 & 7 \\
\hline $3 \mathrm{E}$ & BOC-Pmh-Asn(trt)-Leu-Gly-PheD-Phe-OMe & 2 & 4 & -15 \\
\hline $3 \mathrm{~F}$ & BOC-Pmh-Asn(trt)-Aib-Val-PheD-Phe-OMe & 2 & 4 & -41 \\
\hline $3 \mathrm{~F}$ & BOC-Pmh-Asn(trt)-Aib-Val-PheD-Phe-OMe & 2 & -20 & -63 \\
\hline $3 \mathrm{G}$ & BOC-Pmh-Asn(trt)-Leu-Aib-PheD-Phe-OMe & 2 & 4 & 34 \\
\hline $3 \mathrm{H}$ & BOC-Pmh-AsnD(trt)-Leu-Val-PheD-Phe-OMe & 2 & 4 & -10 \\
\hline $3 \mathrm{I}$ & BOC-Pmh-Asn(trt)-Leu-Val-TyrD(Bn)-Phe-OMe & 2 & 4 & -32 \\
\hline $3 \mathrm{~J}$ & BOC-Pmh-Asn(trt)-Ser(tbu)-Val-PheD-Phe-OMe & 2 & 4 & -28 \\
\hline $3 \mathrm{~K}$ & BOC-Pmh-Asn(trt)-Leu-Ser(tbu)-PheD-Phe-OMe & 2 & 4 & -47 \\
\hline $3 \mathrm{~K}$ & BOC-Pmh-Asn(trt)-Leu-Ser(tbu)-PheD-Phe-OMe & 2 & -20 & -67 \\
\hline $3 \mathrm{~L}$ & BOC-Pmh-Asn(trt)-Leu-Thr(tbu)-PheD-Phe-OMe & 2 & 4 & -49 \\
\hline $3 \mathrm{~L}$ & BOC-Pmh-Asn(trt)-Leu-Thr(tbu)-PheD-Phe-OMe & 2 & -20 & -58 \\
\hline $3 \mathrm{M}$ & BOC-Pmh-Asn(trt)-Thr(tbu)-Val-PheD-Phe-OMe & 2 & 4 & -29 \\
\hline $3 \mathrm{~N}$ & BOC-Pmh-Asp(OBut)-Leu-Val-PheD-Phe-OMe & 3 & 4 & -31 \\
\hline 30 & BOC-Pmh-Asp(OBn)-Leu-Val-PheD-Phe-OMe & 3 & 4 & -40 \\
\hline $3 \mathrm{P}$ & BOC-Pmh-AspD(OBut)-Leu-Val-PheD-Phe-OMe & 3 & 4 & -32 \\
\hline $3 Q$ & BOC-Pmh-Asp(OBut)-LeuD-Val-PheD-Phe-OMe & 3 & 4 & -15 \\
\hline $3 \mathrm{R}$ & BOC-Pmh-Asp(OBn)-LeuD-Val-PheD-Phe-OMe & 3 & 4 & 5 \\
\hline $3 \mathrm{~S}$ & BOC-Pmh-AspD(OBut)-LeuD-Val-PheD-Phe-OMe & 3 & 4 & 10 \\
\hline $3 \mathrm{~T}$ & BOC-Pmh-Asp(OBut)-Leu-ValD-PheD-Phe-OMe & 3 & 4 & -17 \\
\hline $3 \mathrm{U}$ & BOC-Pmh-Asp(OBn)-Leu-ValD-PheD-Phe-OMe & 3 & 4 & -27 \\
\hline $3 \mathrm{~V}$ & BOC-Pmh-AspD(OBut)-Leu-ValD-PheD-Phe-OMe & 3 & 4 & -30 \\
\hline $3 \mathrm{~W}$ & BOC-Pmh-Asn(trt)-LeuD-Thr(tbu)-PheD-Phe-OMe & 3 & 4 & -21 \\
\hline
\end{tabular}




\begin{tabular}{|c|c|c|c|c|}
\hline $3 \mathrm{X}$ & BOC-Pmh-Asn(trt)-LeuD-Ser(tbu)-PheD-Phe-OMe & 3 & 4 & -28 \\
\hline $3 \mathrm{Y}$ & BOC-Pmh-Asn(trt)-Leu-Tle-PheD-Phe-OMe & 3 & 4 & -36 \\
\hline $3 Z$ & BOC-Pmh-Asn(trt)-Leu-SerD(tbu)-PheD-Phe-OMe & 3 & 4 & -24 \\
\hline $4 \mathrm{~A}$ & BOC-Pmh-AspD(OBut)-Leu-Thr(tbu)-PheD-Phe-OMe & 3 & 4 & -42 \\
\hline $4 \mathrm{~B}$ & BOC-Pmh-AspD(OBut)-Leu-Ser(tbu)-PheD-Phe-OMe & 3 & 4 & -42 \\
\hline $4 \mathrm{C}$ & BOC-Pmh-Asn(trt)-Leu-PheD-PheD-Phe-OMe & 3 & 4 & 26 \\
\hline 4D & BOC-Pmh-AspD(OBut)-Val-Tle-PheD-Phe-OMe & 3 & 4 & -12 \\
\hline $4 \mathrm{E}$ & BOC-Pmh-Asn(trt)-Phe-Thr(tbu)-PheD-Phe-OMe & 3 & 4 & -30 \\
\hline $4 \mathrm{~F}$ & BOC-Pmh-Asn(trt)-PheD-Ser(tbu)-PheD-Phe-OMe & 3 & 4 & -23 \\
\hline $4 \mathrm{G}$ & BOC-Pmh-Asn(trt)-Met-Val-PheD-Phe-OMe & 3 & 4 & -28 \\
\hline $4 \mathrm{H}$ & BOC-Pmh-Trp(BOC)-Leu-Val-PheD-Phe-OMe & 3 & 4 & -15 \\
\hline $4 \mathrm{I}$ & BOC-Pmh-Asn(trt)-Leu-Val-ValD-Phe-OMe & 3 & 4 & -34 \\
\hline $4 \mathrm{~J}$ & BOC-DPmh-Asn(trt)-Leu-ValD-PheD-Phe-OMe & 3 & 4 & 14 \\
\hline $4 \mathrm{~K}$ & BOC-DPmh-Asn(trt)-Leu-Val-PheD-Phe-OMe & 3 & 4 & 0 \\
\hline $4 \mathrm{~L}$ & BOC-Pmh-Asn(trt)-Aib-Ser(tbu)-PheD-Phe-OMe & 4 & 4 & -52 \\
\hline $4 \mathrm{~L}$ & BOC-Pmh-Asn(trt)-Aib-Ser(tbu)-PheD-Phe-OMe & 4 & -20 & -77 \\
\hline $4 \mathrm{M}$ & BOC-Pmh-Asn(trt)-Deg-Ser(tbu)-PheD-Phe-OMe & 4 & 4 & -27 \\
\hline $4 \mathrm{~N}$ & BOC-Pmh-Asn(trt)-Tle-Ser(tbu)-PheD-Phe-OMe & 4 & 4 & -30 \\
\hline 40 & BOC-Pmh-Asn(trt)-Gly-Ser(tbu)-PheD-Phe-OMe & 4 & 4 & -18 \\
\hline $4 \mathrm{P}$ & BOC-Pmh-His(trt)-Leu-Ser(tbu)-PheD-Phe-OMe & 4 & 4 & -25 \\
\hline $4 Q$ & BOC-Pmh-His(trt)-Aib-Ser(tbu)-PheD-Phe-OMe & 4 & 4 & -51 \\
\hline $4 Q$ & BOC-Pmh-His(trt)-Aib-Ser(tbu)-PheD-Phe-OMe & 4 & -20 & -68 \\
\hline $4 \mathrm{R}$ & BOC-Pmh-His(trt)-Val-Ser(tbu)-PheD-Phe-OMe & 4 & 4 & -29 \\
\hline $4 \mathrm{~S}$ & BOC-Pmh-Asn(trt)-Leu-Ser(tbu)-TyrD(tbu)-Phe-OMe & 4 & 4 & -50 \\
\hline $4 \mathrm{~S}$ & BOC-Pmh-Asn(trt)-Leu-Ser(tbu)-TyrD(tbu)-Phe-OMe & 4 & -20 & -78 \\
\hline $4 \mathrm{~T}$ & BOC-Pmh-Ser(trt)-Leu-Ser(tbu)-PheD-Phe-OMe & 4 & 4 & -24 \\
\hline $4 \mathrm{U}$ & BOC-Pmh-Asn(trt)-Aib-Thr(tbu)-PheD-Phe-OMe & 4 & 4 & -32 \\
\hline $4 \mathrm{U}$ & BOC-Pmh-Asn(trt)-Aib-Thr(tbu)-PheD-Phe-OMe & 4 & -20 & -26 \\
\hline $4 \mathrm{~V}$ & BOC-Pmh-Asn(trt)-Deg-Thr(tbu)-PheD-Phe-OMe & 4 & 4 & -28 \\
\hline $4 \mathrm{~W}$ & BOC-Pmh-Asn(trt)-Tle-Thr(tbu)-PheD-Phe-OMe & 4 & 4 & -44 \\
\hline $4 X$ & BOC-Pmh-Asn(trt)-Gly-Thr(tbu)-PheD-Phe-OMe & 4 & 4 & -26 \\
\hline $4 \mathrm{Y}$ & BOC-Pmh-His(trt)-Leu-Thr(tbu)-PheD-Phe-OMe & 4 & 4 & -28 \\
\hline $4 Z$ & BOC-Pmh-His(trt)-Aib-Thr(tbu)-PheD-Phe-OMe & 4 & 4 & -44 \\
\hline $4 \mathrm{Z}$ & BOC-Pmh-His(trt)-Aib-Thr(tbu)-PheD-Phe-OMe & 4 & -20 & -66 \\
\hline $5 \mathrm{~A}$ & BOC-Pmh-His(trt)-Val-Thr(tbu)-PheD-Phe-OMe & 4 & 4 & -23 \\
\hline 5B & BOC-Pmh-Asn(trt)-Leu-Thr(tbu)-TyrD(tbu)-Phe-OMe & 4 & 4 & -51 \\
\hline 5B & BOC-Pmh-Asn(trt)-Leu-Thr(tbu)-TyrD(tbu)-Phe-OMe & 4 & -20 & -68 \\
\hline $5 \mathrm{C}$ & BOC-Pmh-Thr(trt)-Leu-Thr(tbu)-PheD-Phe-OMe & 4 & 4 & -27 \\
\hline $5 \mathrm{D}$ & BOC-Pmh-Asn(trt)-Leu-Ser(trt)-PheD-Phe-OMe & 4 & 4 & -41 \\
\hline $5 \mathrm{E}$ & BOC-Pmh-Asn(trt)-Leu-Thr(trt)-PheD-Phe-OMe & 4 & 4 & -36 \\
\hline $5 \mathrm{~F}$ & BOC-Pmh-Asn(trt)-Aib-Ser(trt)-PheD-Phe-OMe & 4 & 4 & -38 \\
\hline $5 \mathrm{~F}$ & BOC-Pmh-Asn(trt)-Aib-Ser(trt)-PheD-Phe-OMe & 4 & -20 & -58 \\
\hline $5 \mathrm{G}$ & BOC-Pmh-Asn(trt)-Aib-Thr(trt)-PheD-Phe-OMe & 4 & 4 & -35 \\
\hline $5 \mathrm{H}$ & BOC-Pmh-Asn(trt)-Ala-Ser(tbu)-PheD-Phe-OMe & 4 & 4 & -45 \\
\hline $5 \mathrm{I}$ & BOC-Pmh-Asn(trt)-Aib-Ser(tbu)-TyrD(tbu)-Phe-OMe & 4 & 4 & -56 \\
\hline
\end{tabular}




\begin{tabular}{|c|c|c|c|c|}
\hline $5 \mathrm{I}$ & BOC-Pmh-Asn(trt)-Aib-Ser(tbu)-TyrD(tbu)-Phe-OMe & 4 & -20 & -79 \\
\hline $5 \mathrm{~J}$ & BOC-Pmh-Asn(trt)-Aib-Ser(tbu)-TyrD(tbu)-Ala-OMe & 5 & 4 & -43 \\
\hline $5 \mathrm{~J}$ & BOC-Pmh-Asn(trt)-Aib-Ser(tbu)-TyrD(tbu)-Ala-OMe & 5 & -20 & -78 \\
\hline $5 \mathrm{~K}$ & BOC-Pmh-Asn(trt)-Aib-Ser(tbu)-TyrD(tbu)-Ile-OMe & 5 & 4 & -19 \\
\hline $5 \mathrm{~K}$ & BOC-Pmh-Asn(trt)-Aib-Ser(tbu)-TyrD(tbu)-Ile-OMe & 5 & -20 & -72 \\
\hline $5 \mathrm{~L}$ & BOC-Pmh-Asn(trt)-Aib-Ser(tbu)-TyrD(tbu)-Phg-OMe & 5 & 4 & -32 \\
\hline $5 \mathrm{~L}$ & BOC-Pmh-Asn(trt)-Aib-Ser(tbu)-TyrD(tbu)-Phg-OMe & 5 & -20 & -80 \\
\hline $5 \mathrm{M}$ & BOC-Pmh-Asn(trt)-Aib-Ser(tbu)-TyrD(tbu)-Val-OMe & 5 & 4 & 0 \\
\hline $5 \mathrm{~N}$ & BOC-Pmh-His(trt)-Aib-Ser(tbu)-TyrD(tbu)-Ala-OMe & 5 & 4 & -36 \\
\hline 50 & BOC-Pmh-His(trt)-Aib-Ser(tbu)-TyrD(tbu)-Ile-OMe & 5 & 4 & 0 \\
\hline $5 \mathrm{P}$ & BOC-Pmh-His(trt)-Aib-Ser(tbu)-TyrD(tbu)-Phg-OMe & 5 & 4 & -30 \\
\hline $5 \mathrm{P}$ & BOC-Pmh-His(trt)-Aib-Ser(tbu)-TyrD(tbu)-Phg-OMe & 5 & -20 & -81 \\
\hline $5 \mathrm{Q}$ & BOC-Pmh-His(trt)-Aib-Ser(tbu)-TyrD(tbu)-Val-OMe & 5 & 4 & 0 \\
\hline $5 \mathrm{R}$ & BOC-Pmh-Asn(trt)-Leu-Ser(tbu)-TyrD(tbu)-Ala-OMe & 5 & 4 & -35 \\
\hline $5 \mathrm{~S}$ & BOC-Pmh-Asn(trt)-Leu-Ser(tbu)-TyrD(tbu)-Ile-OMe & 5 & 4 & 0 \\
\hline $5 \mathrm{~T}$ & BOC-Pmh-Asn(trt)-Leu-Ser(tbu)-TyrD(tbu)-Phg-OMe & 5 & 4 & -34 \\
\hline $5 \mathrm{U}$ & BOC-Pmh-Asn(trt)-Leu-Ser(tbu)-TyrD(tbu)-Val-OMe & 5 & 4 & 0 \\
\hline $5 \mathrm{~V}$ & BOC-Pmh-Asn(trt)-Sp5-Ser(tbu)-TyrD(tbu)-Phe-OMe & 5 & 4 & -11 \\
\hline $5 \mathrm{~V}$ & BOC-Pmh-Asn(trt)-Sp5-Ser(tbu)-TyrD(tbu)-Phe-OMe & 5 & -20 & -60 \\
\hline $5 \mathrm{~W}$ & BOC-Pmh-Asn(trt)-Sp6-Ser(tbu)-TyrD(tbu)-Phe-OMe & 5 & 4 & -11 \\
\hline $5 \mathrm{~W}$ & BOC-Pmh-Asn(trt)-Sp6-Ser(tbu)-TyrD(tbu)-Phe-OMe & 5 & -20 & -42 \\
\hline $5 X$ & BOC-Pmh-His(trt)-Sp5-Ser(tbu)-TyrD(tbu)-Phe-OMe & 5 & 4 & -23 \\
\hline $5 \mathrm{Y}$ & BOC-Pmh-His(trt)-Sp6-Ser(tbu)-TyrD(tbu)-Phe-OMe & 5 & 4 & 0 \\
\hline $5 Z$ & BOC-Pmh-Asn(trt)-Sp5-Ser(tbu)-PheD-Phe-OMe & 5 & 4 & -20 \\
\hline $5 Z$ & BOC-Pmh-Asn(trt)-Sp5-Ser(tbu)-PheD-Phe-OMe & 5 & -20 & -59 \\
\hline $6 \mathrm{~A}$ & BOC-Pmh-Asn(trt)-Sp6-Ser(tbu)-PheD-Phe-OMe & 5 & 4 & 0 \\
\hline $6 \mathrm{~A}$ & BOC-Pmh-Asn(trt)-Sp6-Ser(tbu)-PheD-Phe-OMe & 5 & -20 & -34 \\
\hline $6 \mathrm{~B}$ & BOC-Pmh-Asn(trt)-Aib-Ser(tbu)-PheD-Ala-OMe & 5 & 4 & -42 \\
\hline $6 \mathrm{C}$ & BOC-Pmh-Asn(trt)-Aib-Ser(tbu)-PheD-Ile-OMe & 5 & 4 & 0 \\
\hline $6 \mathrm{D}$ & BOC-Pmh-Asn(trt)-Aib-Ser(tbu)-PheD-Phg-OMe & 5 & 4 & -38 \\
\hline $6 \mathrm{E}$ & BOC-Pmh-Asn(trt)-Aib-Ser(tbu)-PheD-Val-OMe & 5 & 4 & 0 \\
\hline $6 \mathrm{~F}$ & BOC-Pmh-His(trt)-Aib-Ser(tbu)-LeuD-Phe-OMe & 5 & 4 & -47 \\
\hline $6 \mathrm{~F}$ & BOC-Pmh-His(trt)-Aib-Ser(tbu)-LeuD-Phe-OMe & 5 & -20 & -81 \\
\hline $6 \mathrm{G}$ & BOC-Pmh-Asn(trt)-Aib-Ser(tbu)-LeuD-Phe-OMe & 5 & 4 & -45 \\
\hline $6 \mathrm{G}$ & BOC-Pmh-Asn(trt)-Aib-Ser(tbu)-LeuD-Phe-OMe & 5 & -20 & -80 \\
\hline $6 \mathrm{H}$ & $\begin{array}{l}\text { BOC-Pmh-Asn(trt)-AlaD-Ser(tbu)-TyrD(tbu)-Phe- } \\
\text { OMe }\end{array}$ & 6 & 4 & -22 \\
\hline $6 \mathrm{I}$ & BOC-Pmh-Asn(trt)-Gly-Ser(tbu)-TyrD(tbu)-Phe-OMe & 6 & 4 & -13 \\
\hline $6 \mathrm{~J}$ & $\begin{array}{l}\text { BOC-Pmh-Asn(trt)-Ser(tbu)-Ser(tbu)-TyrD(tbu)-Phe- } \\
\text { OMe }\end{array}$ & 6 & 4 & -31 \\
\hline $6 \mathrm{~K}$ & BOC-Pmh-Asn(trt)-Val-Ser(tbu)-TyrD(tbu)-Phe-OMe & 6 & 4 & -26 \\
\hline $6 \mathrm{~L}$ & $\begin{array}{l}\text { BOC-Pmh-Asn(trt)-ValD-Ser(tbu)-TyrD(tbu)-Phe- } \\
\text { OMe }\end{array}$ & 6 & 4 & -15 \\
\hline $6 \mathrm{M}$ & $\begin{array}{c}\text { BOC-Pmh-Asn(trt)-Aib-Ser(tbu)-TyrD(tbu)- } \\
\text { Trp(BOC)-OMe }\end{array}$ & 6 & 4 & -55 \\
\hline $6 \mathrm{~N}$ & BOC-Pmh-Asn(trt)-Aib-Ser(tbu)-PheD(4-NO2)-Phe- & 6 & 4 & -49 \\
\hline
\end{tabular}




\begin{tabular}{|c|c|c|c|c|}
\hline & $\mathrm{OMe}$ & & & \\
\hline $6 \mathrm{O}$ & $\begin{array}{c}\text { BOC-Pmh-Asn(trt)-Aib-Ser(tbu)-TyrD(tbu)- } \\
\text { TyrD(tbu)-OMe }\end{array}$ & 6 & 4 & -58 \\
\hline $6 \mathrm{P}$ & $\begin{array}{c}\text { BOC-Pmh-Asn(trt)-Aib-Ser(tbu)-TyrD(tbu)-PheD- } \\
\text { OMe }\end{array}$ & 6 & 4 & -61 \\
\hline $6 \mathrm{Q}$ & BOC-Pmh-Asn(trt)-Aib-Ser(tbu)-LeuD-PheD-OMe & 6 & 4 & -65 \\
\hline $6 \mathrm{Q}$ & BOC-Pmh-Asn(trt)-Aib-Ser(tbu)-LeuD-PheD-OMe & 6 & -20 & -79 \\
\hline 6R & BOC-Pmh-Asn(trt)-Aib-Ser(tbu)-PheD-PheD-OMe & 6 & 4 & -59 \\
\hline $6 \mathrm{~S}$ & $\begin{array}{c}\text { BOC-Pmh-Tyr(tbu)-PheD-Ser(tbu)-TyrD(tbu)-Phe- } \\
\text { OMe }\end{array}$ & 6 & 4 & -20 \\
\hline $6 \mathrm{~T}$ & BOC-Pmh-Leu-Aib-Ser(tbu)-TyrD(tbu)-Phe-OMe & 6 & 4 & -18 \\
\hline $6 \mathrm{U}$ & BOC-Pmh-Ile-ValD-Ser(tbu)-TyrD(tbu)-Phe-OMe & 6 & 4 & -22 \\
\hline $6 \mathrm{~V}$ & BOC-Pmh-Asn(trt)-Leu-Ser(tbu)-PheD-PheD-OMe & 6 & 4 & -47 \\
\hline $6 \mathrm{~W}$ & BOC-Pmh-Asn(trt)-Aib-Aib-TyrD(tbu)-Phe-OMe & 6 & 4 & -8 \\
\hline $6 \mathrm{X}$ & BOC-Pmh-Asn(trt)-Ser(tbu)-Aib-TyrD(tbu)-Phe-OMe & 6 & 4 & 7 \\
\hline $6 \mathrm{Y}$ & BOC-Pmh-His(trt)-Aib-Ser(tbu)-TyrD(tbu)-PheD-OMe & 6 & 4 & -60 \\
\hline $6 \mathrm{Z}$ & BOC-Pmh-Nap-Aib-Ser(tbu)-TyrD(tbu)-Phe-OMe & 6 & 4 & -25 \\
\hline $7 \mathrm{~A}$ & BOC-Pmh-Asn(trt)-Aib-Ser(tbu)-NapD-Phe-OMe & 6 & 4 & -55 \\
\hline $7 \mathrm{~B}$ & BOC-Pmh-Asn(trt)-Aib-Ser(tbu)-TyrD(tbu)-Nap-OMe & 6 & 4 & -55 \\
\hline $7 \mathrm{C}$ & $\begin{array}{c}\text { BOC-Pmh-Asn(trt)-Aib-Ser(tbu)-TyrD(tbu)-NapD- } \\
\text { OMe }\end{array}$ & 6 & 4 & -60 \\
\hline $7 \mathrm{D}$ & BOC-Pmh-Asn(trt)-Aib-Pro-His(trt)-Phe-OMe & 6 & 4 & -31 \\
\hline $7 \mathrm{E}$ & $\begin{array}{c}\text { BOC-Pmh-Val-Trp(BOC)-Ser(tbu)-TyrD(tbu)-Phe- } \\
\text { OMe }\end{array}$ & 6 & 4 & -23 \\
\hline
\end{tabular}

